34

\title{
EINE EUROPÄISCHE TRANSKULTURELLE UND MEHRSPRACHIGE STADT - RIJEKA UND DIE DEUTSCHSPRACHIGE KULTUR
}

\section{A EUROPEAN TRANSCULTURAL AND MULTILINGUAL CITY - RIJEKA AND THE GERMAN-SPEAKING CULTURE}

\author{
Petra Žagar-Šoštarić, Irvin Lukežić \\ Philosophische Fakultät, der Universität in Rijeka, Rijeka, Kroatien \\ Faculty of Humanities and Social Sciences, University of Rijeka, Rijeka, Croatia
}

\section{Zusammenfassung}

Rijeka/Fiume war im 19. Jahrhundert eine der führenden Industriestädte Europas. Damals entwickelte sie sich, sowohl im Bereich der Politik als auch der Wirtschaft und Kultur. Der bekannte Industrielle aus England beispielsweise, Robert Whitehead, erweitert seine Produktion und entwirft Skizzen für die ersten Torpedoraketen. Es werden die ersten Banken und Handelskammern in Rijeka gegründet. Wohltätigkeitsbälle werden organisiert und Bibliotheken ausgestattet. Wissenschaftler (Naturwissenschaftler), Künstler, Großindustrielle, Politiker nehmen an Debatten teil, um den Ausbau der Stadt zu planen und um die Lebensqualität aller Bürger zu verbessern. Eine direkte Zugverbindung von Wien über Opatija (Abbazia) nach Rijeka (Fiume), mehrere überozeanische direkte Schiffsverbindungen aus Rijeka nach Amerika, Neuseeland und Australien ermöglichen die Erkundung neuer Welten, anderer Sprachen und den Import dergleichen während der Rückkehr eigener Bewohner/Seefahrer oder der Ankunft von Fremdlingen/Migranten als neuen Bewohnern der Stadt. Rijeka/Fiume war und ist es bis heute geblieben: ein europäisches transkulturelles und mehrsprachiges Zentrum. Aus diesem Grund soll die Transkulturalität in diesem Beitrag anhand bekannter Persönlichkeiten deutschsprachiger Herkunft in dieser Stadt veranschaulicht werden. Dieser Beitrag stützt sich und ergänzt die bisherigen Forschungsergebnisse von Ervin Dubrović,

\section{Abstract}

Rijeka/Fiume was one of the leading industrial cities in Europe in the 19th century. At that time, it developed both in the area of politics as well as economy and culture. For example, the well-known industrialist from England, Robert Whitehead, expanded his production and designed sketches for the first torpedo missiles. The first banks and chambers of commerce were founded in Rijeka. Charity balls were organised and libraries were equipped. Scientists (natural scientists), artists, industrial magnates, politicians took part in debates in order to plan the expansion of the city and to improve the quality of life of all citizens. A direct train connection from Vienna via Opatija (Abbazia) to Rijeka (Fiume), several direct oversea boat connections from Rijeka to America, New Zealand and Australia enabled the exploration of new worlds, other languages and the import of the like during the return of residents/sailors or upon the arrival of foreigners/migrants as new residents of the city. Rijeka/Fiume was and remains until today: a European transcultural and multilingual centre. For this reason, the present paper illustrates transculturality using well-known personalities of German-speaking origin in this city. This article is based on and complements previous research results of Ervin Dubrović, Gerhard Dienes and Irvin Lukežić. Aspects of multilingualism/translation as well as multiculturalism are in the foreground of this study. 
Gerhard Dienes und Irvin Lukežić. Dabei stehen Aspekte der Mehrsprachigkeit/Translation als auch Multikulturalität im Vordergrund dieser Untersuchung.

\section{Einleitung}

[...] So wie man durch diese Pforte um die Ecke biegt, zeigt sich ein eben so überraschender als bezaubernder Anblick: die schöne, freundliche, lebhafte Stadt Fiume liegt dem Reisendem gerade zu Füßen, über ihr hängt auf einem Felsenzacken das ehemalige Franzipanische Schloß Tersat, eine noch sehr feste und weitläufige Ruine. Man sieht in Vogelperspektive auf die Gassen der Stadt, und besonders auf die anmuthige, mit Platanenalleen eingefaßte Promenaden, an deren Seite die erst noch ganz zügellose Nymphe der Fiumera so zahm und elegant, zwischen Stein umbordeten Ufern aus feinem Sande dahin flüstert, als ob sie es gar nicht wäre. - Da die Promenade eben auch von einem glänzenden Publikum stark besucht war, so läßt sich denken, welch einen angenehmen Eindruck ein so plötzlicher Übergang von Wildheit und roher Natur zu ausgebildeter Kultur und gefälligem Luxus hervorbringen mußte; ich glaube nirgends können sich diese Extreme schroffer berühren. Über die flachen, schon italienischen Dächer von hohlen, in einander greifenden Ziegeln hinaus, zeigten sich noch in der Fiumera eine Menge von Trabacolis und kleinen Fahrzeugen, dann weiter im Meere [...]. Während in Mersla Vodica die Gewächse noch ganz dieselben sind, wie im nördlichen Ungarn, erblickt man hier gleich den Feigenbaum in freistehendem Wuchs [...] /1/

Bereits Therese von Artner deutete im Jahre 1830 auf ihrer Durchreise nach Italien den transkulturellen Charakter der Stadt Rijeka an und bevor hier das Thema zur Transkulturalität und den Spuren der deutschsprachigen Kultur in Rijeka in diesem Beitrag veranschaulicht wird, bedarf es einer kurzen Begriffserklärung der Transkulturalität nach Wolfgang Welsch für diesen Beitrag.

Welsch /2/ definiert den Begriff Transkulturaliät als ein Zusammentreffen von zweier oder mehrerer unterschiedlicher (auch gegensätzlicher) Kulturen, die zu einer ISSN 1330-0067
Verwischung bzw. Verschwindung von Grenzen führen kann. Es entsteht dadurch zwar keine Globalkultur im Sinne einer einheitlichen Weltkultur, vielmehr entstehen dadurch Kulturen von Individuen und Gesellschaften, welche transkulturelle Elemente in sich tragen. Es ist eine Mischung von unterschiedlichen kulturellen Elementen, entstanden durch verschiedene Herkunft, die ein jedes Individuum und Gesellschaft transkulturell machen. Solche Kulturen entstehen also durch einen Austausch von unterschiedlichen Lebensformen, Lebenseinstellungen, Wertehaltungen und Weltanschauungen. /3/ Am Beispiel der Stadt Rijeka sollen Aspekte der Mehrsprachigkeit/Translation als auch Multikulturalität mit dem Schwerpunkt des Einflusses der deutschen Kultur auf die Entwicklung der Stadt aber auch anhand ihrer durch Erinnerungen geprägten Sicht auf die Stadt Rijeka (notiert in Form von Gedichten, Reiseberichten und fachwissenschaftlichen Beiträgen) in den Vordergrund dieser Untersuchung gerückt werden. Nicht ausgeschlossen sind dabei auch andere Kulturen in Rijeka, welche hier in diesem Beitrag herangezogen werden und die den Ausbau dieser Stadt als einer transkulturellen und mehrsprachigen Stadt unterzeichnet haben.

Erinnert sei an dieser Stelle an Worte Ervin Dubrovićs, der einst zu Rijeka unter dem Titel Grenzen folgendes notiert hat:

[...] Es ist nicht gut, anmaßende Schlussfolgerungen $\mathrm{zu}$ ziehen, aber diese Mauer [gedacht sei an den römischen Limes], die sich tief ins Landesinnere und über Gorski Kotar bis nach Slowenien erstreckte, war eineinhalb Millenien die Ostgrenze des Römischen Reichs. Genau bis Rijeka, genauer bis zu Riječina [gemeint ist der Fluss Fiumara in Rijeka] reichten noch im 18. Jahrhundert die Länder und Provinzen dieses großen Kaiserreichs- Innerösterreich und das

15SN $1330-0067$

Coden: IORME7 
36

Herzogtum Krain. Auf jeden Fall war genau hier anstatt der längst zerstörten Mauer das Flüsschen lange Zeit die Staatsgrenze. Für die einen ist die Grenze völlig unsichtbar, weil auf beiden Seiten die gleichen Menschen wohnen, für die anderen ist sie eine der mythischen Trennlinien, an der die Zivilisation endet und der Balkan beginnt!

Wem eine solche Auffassung von Geografie nicht gefällt, der mag sich daran erinnern, das eine der Grenzen zwischen Ost und West das Flüsschen Leitha ist, das das mitteleuropäische Kaiserreich in Cisleithanien und Transleithanien trennt. Nicht unerwähnt sollen auch die sein, für die der Osten, wie einigen malerischen Vierteln Wiens und Münchens bereits in Triest beginnt! /4/

Geschichtlich betrachtet trafen schon immer unterschiedliche Kulturen, Sitten, Bräuche, Sprachen in dieser Stadt aufeinander, indem die kulturellen Unterschiede weniger sichtbar wurden, weil „auf beiden Seiten, die gleichen Menschen wohnten". Dennoch beschreibt und unterscheidet sehr bildhaft Antun Barac (1894 1955) den Bewohner Rijekas:

Der Fiuman ist eine Rasse für sich, er gehört zu keinem Volk. Er ist eine Mischung von allen, die in diese Handelsstadt gekommen sind, um Handel zu treiben - mit allen und mit allem. Der Fiuman ist Italiener und Jugoslawe - ein Italiener, gezeugt von einem Jugoslawen und großgezogen wie ein Jugoslawe, der aus voller Brust trällert: 'O Italia, o morte!', und ein Jugoslawe, ein stilles, ängstliches Tier, das wegen seiner Interessen seine nationale Herkunft hinter einem neutralen, ausdruckslosen Händlerlächeln verbirgt.

Und Riječanin, Recan, Ričanin - das ist die Masse der Namenlosen, die nicht gefragt wird, eine quantité négligeable, das sind die Bewohner der Arbeiterhäuschen, Keller und Dachböden, die Knechte und Tagelöhner, die kleinen Handwerker und Hilfsarbeiter, eine Masse, die nur einen Kopf hat und vielleicht von nur einem Schlag fallen wird." /5/

Die Stadt Rijeka bzw. Fiume war bereits im 19. Jahrhundert eine äußerst wichtige europäische Hafen- und Handelsstadt. Transportiert wurden unterschiedliche Waren vor allem nach Amerika und zahlreiche Menschen migrierten ISSN 1330-0067 über diese Stadt in die versprochen Welt - Amerika. /6/

Industrielle aus England und Italien, aber auch aus anderen Städten Europas kommen im 19. Jahrhundert in die Stadt am Flaum/ Fiume/ Rijeka, um sich geschäftlich in dieser Hafenstadt zu etablieren. Gesprochen wurde sowohl Ungarisch, Italienisch, Deutsch, Slowakisch, Kroatisch, Tschechisch und Slowenisch. Ein einfacher Bürger (der Arbeiterklasse) beherrschte mindestens drei Sprachen.

Rijeka ist im 19. Jahrhundert eine wichtige Industriestadt Europas. Sie erlebt zu dieser Zeit ihre Blütezeit, sowohl im Bereich der Politik als auch der Wirtschaft und Kultur. (Sogar Sigmund Freud, Otto und Hans Gross sowie Cesare Lombroso besuchten öfter diese Stadt und ihre Umgebung.)

Der bekannte Industrielle aus England, Robert Whitehead, erweitert seine Produktion und entwirft Skizzen für die ersten Torpedoraketen. Arthur Steinacker, ein deutscher Intellektueller gründet in Rijeka Zuckerraffinerien und Banken. Eine Frankfurterin, Natalia Ritter, wurde zur ersten Dame von Rijeka, da sie die Ehefrau des ersten Rijekaner Bürgermeisters wurde.

Mit den Anfängen der Industrialisierung und Technologisierung der Stadt, die unter gemeinsamer Wirkung unterschiedlicher Kulturen und Sprachen ausgebaut wurde, wurden auch die ersten Hotels für Migranten gebaut. Sowohl für diejenigen, die in Rijeka antrafen und sesshaft wurden als auch für all diejenigen, die aus Rijeka oder über Rijeka weiter über den Atlantik reisten und auf der Suche nach einer besseren Zukunft waren.

Mit der britischen Cunard Line Company/Gesellschaft bekommt Rijeka beispielsweise eine direkte Verbindung mit New York (den USA). Rijeka wurde durch die zahlreichen Zuwanderungen aber auch Auswanderungen nach Amerika eine rege Transitstadt und ein transkulturelles Zentrum der Mehrsprachigkeit /7/. Im Jahre 1903 fasst die Tageszeitung Novi List das Migrationsproblem in Rijeka zusammen, in dem es Notiz machte, dass in einem Tag mehr als 199 Menschen Rijeka für Amerika verließen. Dort haben sie Arbeit gefunden, indem sie, wie wir anhand der Untersuchungen von Dubrović erfahren, als Minenarbeiter, Waldarbeiter und in Fabriken gearbeitet haben. Viele arbeiteten

Coden: IORME7 
mit Holz (Holzfässer für Whiskey) andere wiederum auf Plantagen oder erbauten die ersten Wolkenkratzer /8/.

In den Jahren um 1870 wurde der Hafen Rijekas transformiert, indem sich der Transport nicht nur auf Passagiere fokussierte, sondern auch auf den Cargotransport von Waren, die in der Stadt (und Umgebung) produziert wurden, wie: Holz, Salz, gesalzenes Fleisch, Tabak, Zucker, Seide und Schokolade. Eine direkte Zugverbindung von Wien über Opatija (Abbazia) nach Rijeka und weiter ermöglichte die Entwicklung des Tourismus auf diesem Gebiet und trug zur Gründung des ersten Hotels Villa Angiolina bei. Das Hotel gründete die Familie Scarpa (Großgrundbesitzer und Industrielle aus Italien) im heutigen Opatija (früher Abbazia) direkt am Meer für Freunde.

Im Zusammenhang mit dem elitären Freundeskreis der Familie Scarpa werden zwei wichtigen Namen in diesem Beitrag herangezogen und dargestellt, die u.a. die Stadt Rijeka in jeder Hinsicht beeinflussten. Es geht dabei um 1. Natalia Ritter de Záhony: eine Dame, die in zahlreichen Dokumenten und verfügbaren Forschungsunterlagen eher im Kontext mit ihren Männern erwähnt wird als wegen ihrer Person selbst und 2. geht es um Arthur Steinacker, einem erfolgreichen Fabrikanten und Bankier. Es geht dabei um Funde, die wir versucht haben, systematisch zusammenzustellen, sowohl hinsichtlich Natalia Ritter als auch Arthur Steinacker. Hinter beiden Namen steht eine langjährige Forschungsarbeit, die wegen einer großen Anzahl an bisher unerforschten (in deutscher Sprache verfassten) Dokumenten noch lange nicht abgeschlossen ist und künftig Archiv- und Forschungsarbeit fordern und neue interdisziplinäre Forschungsbereiche den Germanisten öffnen.

Was den Namen Natalia Ritter de Zahony angeht, so ist anhand zahlreicher aus den Archiven entnommenen Dokumenten ans Licht gekommen, dass es sich für die Entwicklung der Stadt bedeutsamen deutschen Spur in Rijeka handelt. Diese war auf dem Gebiet der Sprache, Kultur und Literatur wichtig und wird hier im Weiteren aus unterschiedlichen Aspekten veranschaulicht.

\section{Natalia Ritter (de Záhonys) und die Stadt Rijeka}

Natalia Ritter de Záhony war zweimal mit äußerst einflussreichen Männern verheiratet. Beide waren ehemalige österreichische Offiziere und wichtig im Militär- als auch im Zivilbereich. Beide hatten sehr gute Beziehungen zur damals wohlhabenden Adels- und gehobenen Bürgergesellschaft und setzten sich für den gesellschaftlichen Fortschritt und das Wohlergehen der Bürger in Rijeka ein. Natalia Ritter de Záhony (später Natalia Ciotta) ist vor allem im Kontext ihrer beiden Ehen der Öffentlichkeit bekannt. Als Schriftstellerin, Übersetzerin, Wohltäterin ist sie unbekannt und es ist wenig Notiz bisher genommen.

Natalia Ritter ist am 25. Dezember 1831 in Gorizia, damals Görz, geboren /9/. Sie entstammt einer sehr reichen, vielköpfigen deutschen Familie, welche noch vor Natalias Geburt in den niederen Adelsstand erhoben wird. Den Urkunden und Archiven zufolge ist der Nachname Ritter im Jahre 1533 zum ersten Mal erwähnt. Die Familie, eine erfolgreiche Handels- und Unternehmerfamilie, kommt ursprünglich aus Frankfurt am Main und gehörte der evangelischen Kirche an /10/.

Ende des 19. Jahrhunderts befanden sich einige der Familienmitglieder Ritter im benachbarten Österreich und Tschechien. Auch dort war die Familie sehr erfolgreich im Bereich des Handels, des Bankwesens und der Industrie.

Im Jahre 1829 wurde Natalias Vater, Johann Christian Ritter, mit dem Adelsnamen de Záhony ausgezeichnet, so verschaffte er der Familie die Zugehörigkeit zum ungarischen Adel /11/.

Christian Johann Ritter de Záhony zeugte ganz im Sinne des protestantischen Glaubens eine große Familie. Mit seiner ersten Ehefrau Maria Bressiach, eine angeblich aus Bakar stammende wohlhabende Dame, bekam er acht Kinder /12/. Nach ihrem Tode heiratete er die fünfzehn Jahre jüngere Amalia Hoffman aus der Schweiz. In dieser Ehe zeugte er sechs weitere Kinder. Natalia war das dreizehnte und vorletzte Kind. Nach dem Tod ihres Vaters siedelte Natalia mit ihrer Mutter aus Görz nach Wien, wo sie ihre 
38

Kindheit verbrachte. Im Sanatorium für Orthopädie Jakob Heine im Jahre 1843 lernte sie die erstgeborene und frühverstorbene Tochter, Emily Bulwer Lytton, des bekannten Schriftstellers und Politikers Edward Bulwer Lytton kennen $/ \mathbf{1 3} /$.

Mit neunzehn Jahren lernte Natalia Ritter de Záhony den Rijekaner Pietro Scarpa kennen und wurde in Rijeka 1850 zu seiner Ehefrau. Pietro Scarpas Vater war der reichste Industrielle und Schiffswerftinhaber in Rijeka derzeit. Er war u.a. Vorsitzender der Handelsfirma Paolo Scarpa und Angiolina Sartorio in Triest /14/. Sein Industrieimperium wuchs und so wurde er zum Vorsitzenden der Handelskammer und Gründer der Metallindustrie als auch Inhaber einer Handelsfirma und Vizekonsul des Königreichs Dänemark in Rijeka. Angiolina Scarpa, Pietros Mutter aus Triest, war Tochter des reichen Patriziers Sartorio aus San Remo, der in Rijeka verstarb /15/.

Als Vorsitzender eines großen Industrieimperiums in Rijeka übte Pietro Scarpa /16/ zahlreiche zusätzliche Funktionen aus. An dieser Stelle seien nur einige hervorgehoben wie: Vorsitzender der Aktiengesellschaft der Fonderie metalli, Vorsitzender der Societá industriale triestino-fiumana und Vorsitzender des Handels- und Seewesensrechts des Kreisgerichts in Rijeka. Pietro Scarpas weitere Vorhaben wurden wegen einer seltenen und tödlichen Krankheit Fungo mendulare (Pilzkrankheit) abgebrochen. Die Gründung der Kreditbank (Credito Mobilare) wurde durch seinen frühen Tod nicht realisiert. Mit nur neunundzwanzig Jahren blieb Natalia verwitwet und beschloss im Jahre 1863 eine zweite Ehe einzugehen. Sie heiratete Giovanni de Ciotta, der später der erste Bürgermeister von Rijeka wurde. Giovanni de Ciotta war Sohn des reichen und einflussreichen Kaufmanns Lorenzo de Ciotta aus Livorno, der in seine Heimatstadt Rijeka mit der Absicht zurückkehrte, diese Stadt zu einer modernen mitteleuropäischen Stadt auszubauen. Damals war Rijeka ein reges gesellschaftliches und kulturelles Zentrum. Straßen wurden gebaut. Es wurde der Verkehr reguliert, das Abflusssystem eingeführt und der Markt ausgebaut. 1885 bekommt auch das Nationaltheater ein repräsen- tatives Gebäude und die Stadt wurde im gleichen Jahr zum ersten Mal mit Strom versorgt./17/

Natalia Ritter de Záhony, jetzt Natalia Ciotta, Ehefrau des ersten Bürgermeisters von Rijeka, hatte - und dieses ist hervorzuheben - vor ihrer Eheschließung und der Ernennung ihres Gatten zum Bürgermeister, ein aktives gesellschaftliches Leben geführt. Sie organisierte in Rijeka diverse Veranstaltungen und Empfänge, in denen Spenden für obdachlose, elternlose Kinder oder finanziell schwächere Familien gesammelt wurden.

Im Jahre 1867 gründete sie die Pick-nick Gesellschaft. Es war eine Gesellschaft die der in Triest seitens Heinrich von Littrow gegründeten Schiller-Gesellschaft ähnelte. Einen feierlichen, in Versen verfassten Prolog zur Eröffnung des Pick-nick-Saales lieferte als aktives Mitglied am 25. Juli 1867 selbst der Hafenkapitän Heinrich von Littrow /18/. Laut La Bilancia 1971 wurden im Rahmen der Pick-nick-Gesellschaft, Lesungen und Vorstellungen organisiert /19/. So hielt Professor Ferdinand Wenedikter einen Vortrag zu Heinrich von Kleists Schaffen und Werk. Heinrich von Littrow sprach zum Thema unter dem Titel Die Ästhetik der Lebenskunst. Im Mai des gleichen Jahres wurden zwei deutsche Komödien Das erste Mittagessen und Nach Mitternacht aufgeführt /20/.

Wie bereits erwähnt blieb Natalia bis heute in literarischer Hinsicht der Öffentlichkeit unbekannt. In der Universitätsbibliothek Rijeka und vorrangig anhand der Forschungsarbeit von Irvin Lukežić sind die ersten Informationen über ihr Werk und Schaffen aufgefunden worden. Ihre Werke, wie wir es bisher nachvollziehen konnten, schrieb sie auf Deutsch und derzeit (Stand Juli 2020) wissen wir, dass folgende bisher vorkommende Titel aus ihrer Feder stammen: Ausser dem Geleise (Roman), Vom Markte des Lebens (Roman) und Geschichte eines Regenmantels. Diese Werke wurden teilweise in den damaligen Wiener und Berliner Tagesblättern veröffentlicht. Der zweibändige Roman Metamorphosen I und II ist im Zeitungsblatt Pester Lloyd in Form eines separaten Buches 1878 in Budapest erschienen /21/.

Unter dem Pseudonym R. Enze veröffentlichte Natalia ihre restlichen Werke, die bisher nicht aufgefunden worden sind. Bekannt ist, dass 
zwischen 1872 und 1873 ihr Roman Lotta senza Gloria ins Italienische übersetzt und im Tagesblatt La Bilancia veröffentlicht wurde. Der Übersetzer des Romans (höchstwahrscheinlich aus dem deutschen Original übersetzt, welches derzeit nicht aufzufinden ist) ist unter den Initialen E.B. bekannt. Beide Buchstaben weisen auf den Publizisten und Beamten Ernesto Brelich hin /22/. Bekannt ist Brelich auch anhand der Übersetzungen der Werke des ungarischen Literaten Móra Jókaia /23/. Das Blatt La Bilancia 1878 fasst mit folgenden Worten die Erscheinung des zweibändigen Romans fest:

La nobilissima signora Natalia de Ciotta, consorte al nostro podestá, fa pubblicare, nelle apendici del reputato giornale il Pester Lloyd, un nuovo suo romanzo, postante il titolo: Le metamorfosi. Notiamo che l'egregia scrittrice pubblicó in passato, sotto il speudonimo R.Enzo, altre due romanzi Lotta senza gloria e Su falsi sentieri il primo die quial venn pure volto in idioma italiano e pubblicato nelle nostre appendici l'anno 1872. Il pubblico tedesco e la critica hanno accolto con molto congratulazioni per la nuova oper in corso di pubblicazione, che siamo certi, verrá agradita a somiglianza di quelle che la precedere. /24/

1888 erschien Natalias Reisebericht Im Land der Pharaonen, der u.a. auch in italienischer und ungarischer Sprache veröffentlicht wurde /25/. Sie schrieb ihre Werke vorwiegend auf Deutsch aber auch auf Italienisch und Ungarisch. Unklar ist noch derzeit, ob und welche Werke sie selbst sowohl ins Ungarische als auch ins Italienische übersetzte.

In der damals einzigen und bis heute bekannten Tageszeitung aus Rijeka La Voce del popolo, erschien 1910 ein Artikel unter der Rubrik Litterati fiumani, in dem u.a. steht, dass Natalia de Ciotta lesenswerte Romane auf Italienisch und Deutsch publizierte /26/.

Aufgrund der Angaben aus der ungarischen Enzyklopädie Magyarország vármegyéi és városai verfügen wir heute über die Angabe, dass Natalia in Rijeka gestorben ist /27/. Mit 63 Jahren, am 19. Mai 1895 um 7.00 Uhr verstarb sie an einer schweren Krankheit, worüber ihr Arzt Dr. Juraj (Giorgo) Catti berichtete.

ISSN 1330-0067
Das Leben und Wirken Natalia Ritter de Záhonys, später Natalia Ciottas, haben wir aufs Neue entdeckt, doch das Erforschen ihres literarischen Werkes steht noch bevor. Ihre Werke unter dem Titel Lotta senza Gloria, Ausser dem Geleise (Roman), Vom Markte des Lebens (Roman) und Geschichte eines Regenmantels konnten bisher nicht aufgefunden werden, doch anhand von Sophie Patakys Zusammenstellung erschienener Werke weiblicher Autoren seit 1840 (aus dem Jahre 1898), wurde sichtbar, dass der Roman Die Geschichte eines Regenmantels vorhanden und anscheinend auch zu einer gewissen Zeit gelesen bzw. bekannt war /28/. Es bedarf an dieser Stelle eine Fortsetzung weiterer und mehrschichtiger, interdisziplinärer Forschungsarbeit im kulturgeschichtlichen Sinne, die eine tiefgehende Analyse unter literatur-/sprachwissenschaftlichen Aspekt der Germanisten ergänzt werden könnte. In dieser Hinsicht ist dieser Beitrag ein wichtiger Ausgangspunkt für weitere Forschungsschritte hinsichtlich der Erkundung deutscher Spuren (der deutschen Sprache, Literatur und Kultur) auf diesem Gebiet $/ 29 /$.

\section{Arthur Steinacker und Rijeka}

Was die bereits erwähnte Person Arthur Steinacker angeht, so war er seiner Herkunft und Weltanschauung nach Deutscher, der in den 1890 Jahren vor allem ein angesehener Bankier in Rijeka und Triest war.

Geboren ist er als Pastorensohn in der heutigen Slowakei, einstiges Ungarn, am 21. Oktober 1844 in Göllnitz/Gelnica/Gölnicbanya unfern von Košice. Später siedelte seine Familie nach Weimar. Trotz seiner deutschen Wurzeln war sein Leben und Schaffen von der ungarischen Kultur und Lebensweise geprägt. Der Großvater Arthur Steinackers, Gabriel Wilhelm Steinacker (1743-1799), um an dieser Stelle die transkulturelle Familiengeschichte auszuholen, ist in Halle geboren und gründete in Dessau einen Lesezirkel und Leihbibliothek. Arthurs Vater, Gustav Steinacker ist in Wien geboren. Mit seiner Familie zog er nach Pest/Ungarn. In Buttelstadt gründete er später den Fröbelverein (1859) und veröffentlichte ein Buch unter dem Titel Bilder, Studien und Klänge aus dem Bereich 
40

des Elternhauses und Kindergartens der Bewahranstalt und Volksschule. /30/ Unter dem Pseudonym G. Treumund veröffentlichte er in Leipzig das Werk unter dem Titel Herzenklänge. Ausgewählte Dichtungen eines Deutschungarns /31/. Zu dieser Zeit (1846) lebte die Familie Gustav Steinackers schon in Triest. Später zogen sie wieder nach Weimar und im Jahre 1861 nach Rom /32/. Die Steinacker-Familie gehörte der gehobenen Bürgerklasse. Sie gehörten sowohl dem Bildungsbürgertum als auch später dem Besitzbürgertum /33/. Verglichen kann seine Lebensweise und Herkunft mit Namen großer Schriftsteller (evangelischer Abstammung) werden, wie: Lessing, Herder, Wieland, Lichtenberg, Hölderlin, Fichte, Schelling etc. Sein Vater, Gustav Steinacker, lernte Richard Wagner 1852 in Zürich während einer Vorlesung über den Ring der Nibelungen kennen. Befreundet war die Familie eng mit Eduard Genast, dem deutschen Sänger, Schauspieler, Komponisten und Theaterdirektor in Weimar. Franz Liszt beispielsweise gab Arthurs Schwester, Irma Steinacker (verheiratet mit dem bekannten Carl von Radecki), Klavierunterricht in Weimar. Liszt und seine damalige Lebensgefährtin Caroline zu Sayn-Wittgenstein zählten zum Freundschaftskreis der Familie. Wie aus unterschiedlichen Dokumenten sichtbar, lebte die Familie Steinacker im Hause von Ottilie von Goethe (der Ehefrau des Sohnes von Goethe, August von Goethe) in Weimar.

Seine Laufbahn als Bankangestellter begann Arthur zunächst in Stuttgart, danach baute er seine Karriere im Bankwesen erfolgreich in London auf. Einige Jahre später zog er nach Paris und kehrte danach wieder nach Ungarn und wieder nach England/London zurück. Aus London besuchte er diverse Städte in Indien, Triest, Budapest und Rijeka. Rijeka wurde zu seinem Wohnsitz, wo er zum englischen Botschafter ernannt wurde. Als belgischer Vizekonsul war er zu der Zeit bereits ernannt. Anhand seiner Arbeitserfahrungen und Weltansicht baute er gute Verhältnisse zwischen Rijeka, London, Budapest und Wien auf. Zuvor war er der belgische Vizekonsul in Budapest. Aus Dokumenten ist bekannt, dass er in Rijeka, Ende der 1880er Jahre, die Ungarische allgemeine Kreditbank mit dem Sitz in Budapest, als Zweigstelle in Rijeka, gründete und im gleichen Jahr laut dem Tagesblatt La Bilancia, trat er mit Irma (seiner Schwester), die in Rijeka bereits eine bekannte Pianistin/Chemballistin war, im Gouverneurspalast in Rijeka auf. Gespielt wurde Trio in DMoll des bekannten Komponisten Felix Mendelssohn Bartholdy. Im gleichen Jahr gründete Arthur seine Firma in Rijeka SteinackerEComp. Diese beschäftigte sich mit Finanzangelegenheiten, Import, Export und Anleihen. Die später gegründete Kreditbank führte er gemeinsam mit dem in Frankfurt am Main geborenen Baron Anselm von Totschield. Totschield kam dienstlich nach Rijeka und laut Lukežićs Untersuchungen mietete er 1879 die Räumlichkeiten des Hotels Europa in Rijeka zu Arbeitszwecken./34/ Arthur Steinacker gründete weitere Fabriken, wie die Zuckerfabrik, später auch die Reisfabrik in Rijeka. Es handelte sich dabei um die Fiumaner erste ungarische Reisschäl- und Reisstärke-Fabrik, deren Generalstellvertreter die Firma Steinacker und Co. Fiume war. Aus diesen Fabriken wurde u.a. Reis und Zucker, die wir bereits am Anfang dieses Beitrags erwähnt haben, über den Hafen in Rijeka nach Amerika transportiert.

Als Mitglied der Rijekaner Handelskammer war er äußerst aktiv und bewirkte die Gründung von anderen Firmen (weiteren Zuckerraffinerien), Sport und Kulturhallen, wie auch das Einrichten von öffentlichen Innen-und Außenräumen in Rijeka. Unter seiner Leitung wurde das Rijekaner Kasino eröffnet (Fiumei Lloyd), ein Reiterklub, und Wohltätigkeitsbälle wurden organisiert. Es ist anzunehmen, dass er diese höchstwahrscheinlich gemeinsam mit Natalia Ritter organisierte. Ein Beweisdokument dazu gibt es bis Dato nicht.

Zusammengearbeitet hat Steinacker auch mit Wilhelm Singer (dem Nähmaschinen Magnat), der Rijeka 1884 verließ und nach Wien zog. Der Rijekaner Schiffsinhaber Kazimir Kozulić, ernannte Arthur zum Vorstandsmitglied in der Rolle des Direktors für seine Firma.

Am 9. März 1893 heiratete Arthur die aus Leipzig stammende Elise Dressler. Der bekannte Architekt Giovanni Rubinich erbaute für die Familie eine Villa im Rijekaner Stadtteil Belveder. 1894 gründete er bereits die Schiffswerft Societá di costruzioni navali Howaldt $\mathcal{E}$ Co. Gemeinsam mit Rudolf Kinski aus Wien 
gründete er ein Jahr später (1895) die Internationale Elektrizitäts-Gesellschaft in Rijeka, vertreten durch Max Karl. Es handelte sich dabei vor allem um den Ausbau einer kleinen Dampfbahn über Matulji nach Opatija bis hin nach Lovran. Hervorzuheben sei an dieser Stelle, das bereits im Jahre 1877 eine poetische Beschreibung dieser Ortschaften aus der Feder Heinrich von Littrows im Gedicht Matuglie (Matulji) erschien /35/:

Die Inseln Veglia, Cherso und die Küste Von Istrien, mit ihren dunkeln Grün, Volosca, Abbazia, Ika und Lovrana, Wo Myrthe, Lorbeer und Kastanie blüh'n.

Arthur Steinacker hielt auf der Reise mit der sibirischen Bahn nach China im Jahre 1902 seine viermonatigen Eindrücke im Reisebericht unter dem Titel Aufder sibirischen Bahn nach China fest. Danach kehrte er wieder nach Rijeka zurück, um 1910 die Rijekaner Industrie- und Handelsgesellschaft Fluminense zu gründen. Dank seines Engagements wurde auch die Schokoladenfabrik gegründet. Sehr engagiert war er auch im Rahmen der Gründung der Konzertgesellschaft und der Freimaurerloge Sirius in Rijeka.

Zu Beginn des Ersten Weltkrieges hat er Rijeka verlassen und zog zurück nach Stuttgart, wo er in seiner Jugend als Bankangestellter seine Karriere angefangen hat. Bis heute ist es unklar, wieso er Rijeka verlassen hatte. Wir sind der Annahme, dass die Kriegszeit höchstwahrscheinlich viele seiner Pläne zu Nichte gemacht hatte und zu bedenken wäre an dieser Stelle, sein bereits fortgeschrittenes Alter. Am 20. Februar 1915 starb er im Marienhospital in Stuttgart. Doch die Villa Steinacker auf Belveder und andere imposante Bauten aus seiner Zeit stehen noch heute pompös zwischen den neueren Bauten in Rijeka. Sein Vermögen war imposant und befand sich sowohl in Stuttgart, Rijeka (zwei Villen und zahlreiche Firmen), Tirol (Wälder am Fuße der Zugspitze), Montfalcone, Triest und Tokai (Weingüter und Häuser).

Anhand der bisher untersuchten Unterlagen ist festzuhalten und zu schließen, dass sich Steinacker europaweit engagiert und überall wohlgefühlt hatte, denn wie wir aus den Lebenserinnerungen (1937) seines Bruders erfahren, waren ihre Heimaten, sowohl das heutige Ungarn, die ISSN 1330-0067
Slowakei, Italien, Deutschland, Österreich, England und für Arthur letztendlich Rijeka. Edmund Steinacker, sein Bruder, hielt seine Rijeka-Aufenthalte folgendermaßen in Erinnerung:

Im Januar 1882 machte ich den Ausflug einer Gruppe von Reichstagsabgeordneten zur Besichtigung der Fiumaner Hafenbauten mit und verband damit einen Besuch meines Bruders Arthur, der zwei Jahre vorher in diese von Ungarn nach Möglichkeit geförderte einzige Hafenstadt des Landes übersiedelt war. Wir wurden glänzend empfangen. Der Gouverneur Graf Szapárn gab uns ein Diner und am Abend des nächsten Tages eine Soirée mit überaus interessanter Gesellschaft. [...] Der Hafenbau, der allerdings an den größeren Verhältnissen des österreichischen Triest gemessen sich noch in bescheidenen Verhältnissen bewegte, gab mehr ein Zukunfts- als ein Gegenwartsbild, aber es zeigte sich auch allen Richtungen eine aufstrebende Entwicklung der Stadt, die durch die Errichtung der Whiteheadschen Torpedofabrik ein Interesse für alle Kriegsmarinen der Welt bot. Wir wohnten der Probelancierung eines Torpedos nach einem in der Bucht verankerten Ziele bei und bewunderten die sinnreiche Konstruktion dieser gewaltigen Seekriegswaffe. Am Nachmittag des zweiten Tages wurde zu einer Fahrt nach Abbazia, diesem durch den Generaldirektor Schüler der Südbahn geschaffenen Kurort, benutzt, wo wir nach der winterlichen Fahrt durch die ungarischen Ebene und den trostlos kahlen Karst uns fast in den Frühling versetzt fühlen konnten. [...] /36/

Es ist ein großer Verlust, dass Arthur Steinecker selbst keine Lebenserinnerungen aufgezeichnet hatte. Diese würden viele unserer Forschungslücken zur Präsenz der deutschsprachigen Kultur in Rijeka und Umgebung (dem Kvarner) decken und ergänzen können. Erinnern wir uns an dieser Stelle abschließend an Heinrich von Littrows literarische Erinnerung an Rijeka im Gedicht Fiume:

Liburnien hiess das Land im Alterthume, Im Bau der Schiffe war das Volk bewandt, Die Flotte baute Ceasar sich zu Ruhme 
42

Die gegen Markus-Antonius er gesandt, Und dieses Gewerbe hat sich auch erhalten, Die Jungen bauen rüstig, wie die Alten. /37/

\section{Schlussfolgerung}

Man könnte an dieser Stelle der Frage nachgehen, inwieweit Gedichte (Heinrich von Littrows), literarische Werke (Natalia Ritters), Reiseberichte (Arthur Steinackers und Therese von Artners), Lebenserinnerungen (Edmund Steinackers) als literatur-historisches Genre der Frage der Transkulturalität hinsichtlich der Stadt Rijeka nachgehen. In diesem Beitrag ist bereits der Versuch unternommen, einen Anfang zu weiteren Untersuchungsaspekten der Transkulturalität am Beispiel der deutschsprachigen Kultur in Rijeka zu legen, denn Transkulturalität ist nach Wolfgang Welsch, wie bereits am Anfang erwähnt, kein neues Phänomen, sondern es ist eine historische Genese, welche die "Homogenitätsfunktion und die separatistische Vorstellung von Kultur" /38/ durchdringt, die gestützt an der historisch bestehenden Möglichkeit einer transkulturellen Kommunikation in einem kulturellen $\mathrm{Ne}$ tzwerk ist und welches hier am Beispiel der Präsenz der deutschsprachigen Kultur in Rijeka veranschaulicht wurde.

Es ist in diesem Beitrag anhand langjähriger und noch längst nicht abgeschlossener Forschungsarbeit der Versuch gewesen, zu zeigen, wie Reflexionen der deutschsprachigen Kultur auf kulturell Fremdes (hinsichtlich der Stadt Rijeka) im Zeitgeist einer gewissen Zeitspanne (Epoche) präsentiert und vorhanden waren.

Mit diesem Beitrag soll ein Anfang gelegt sein, für weitere transkulturelle Forschungsvorhaben zur Geschichte, Sprache, Kultur, Literatur, Translation und transkulturelle Kommunikation nicht nur in Bezug auf die deutschsprachige Kultur in Rijeka, sondern allderjenigen Kulturen und Sprachen die in einer Zusammenarbeit, eine transkulturelle Stadt erbaut haben, deren Geschichte längst vergessen und doch überall in der diesjährigen europäischen Kulturhauptstadt 2020 präsent ist, wie Worte von Giacomo Scotti es einst im Gedicht Ich möchte eine Stadt sein bestätigt haben:

[...] Ich möchte eine Stadt sein, die lächelt wegen des Lächelns, das uns ein eiliger Fußgänger schenken kann.

Eine Stadt mit dem Besten

Einer jeden erdenklichen Stadt

[...]

mit Plätzen, Märkten und breiten Straßen.

[...]

Eine Stadt mit einer Seele, ein Festtag für Gedanken ohne Zorn und Wut, bedächtig, lesbar in jeder Sprache[...] /39/

Notes

/1/ Artner v., Therese (1830). Briefe über einen Theil von Croatien und Italien an Caroline von Pichler. Augsburg. S. 108-109 (Louisenstraße). Therese von Artner wurde am 19. April 1772 in Šintava in der Slowakei geboren und starb am 25. November 1829 in Zagreb. Sie schrieb Theaterstücke und Lieder. Am bekanntesten ist ihr Epos Die Schlacht von Aspern. Sie kam 1822 nach Zagreb und setzte dort ihre literarische Arbeit fort. In Deutschland war sie bereits 1789 eine bekannte Autorin. In ihrem Reisebericht unter dem Titel Briefe über einen Theil von Croatien und Italien und Caroline beschrieb sie sehr akribisch und ganz im Geiste der deutschen Romantik ihre Eindrücke von der Reise aus Zagreb über Rijeka und weiter nach Italien. Es ist erwähnenswert, dass Therese von Artner eine der ersten Autoren von Reiseberichten ist, deren literarisches Werk den Wert von archäologischen Stätten hervorhebt und diese zu schützen sucht. Vgl. dazu weiter Žagar-Šoštarić, Petra/Lukežić, Irvin (2020): Tragovima kulture njemačkog govornog područja u Rijeci. (derzeit noch unveröffentlicht)

/2/ Wolfgang Welsch (2009). Was ist eigentlich Transkulturalität? In: Lucyna Darowska, Claudia Machold (Hg.): Hochschule als transkultureller Raum? Beiträge zu Kultur, Bildung und Differenz. Transkript, Bielefeld. S. 39-67.

/3/ Vgl. weiter dazu Welsch (2009). Op.cit., S. 39-42.

/4/ Dubrović, Ervin (2020). Grenzen. In: Vijenac. Novine Matice hrvatske za književnost, umjetnost i znanost. Nr. 632. Hier zitiert nach: Dienes M. Gerhard (2020) (Hg. et.al). Europa erlesen Rijeka. Wieser Verlag. Klagenfurt. S. 19-24. (Übersetzt von Gero Fischer).

/5/ Barac, Antun (2009). Fiume. Flüchtige Eindrücke. In: Bačić-Karković, Danijela (Hg): Rijeka u Priči: Hrestomatija književnih tekstova o gradu na Rijeci. Filozofski fakultet. Rijeka. S. 211-214. Hier zitiert nach Dienes, Wolfgang (2020) (Hg. et.al). Europa erlesen. Wieser Verlag. Klagenfurt. 28-29. 
/6/ In Rijeka wurde seitens des Museums der Stadt Rijeka im Jahre 2012 eine Ausstellung organisiert und zweisprachige Publikation unter dem Titel From Central Europe to America 1880-1914 herausgegeben. Zum Thema Auswandern und Migration haben mehr als 30 Wissenschaftler aus Europa und den USA teilgenommen, um diese Ausstellung und Monographie gestalten zu können. Auch sei an dieser Stelle betont, dass fortan im Beitrag der Begriff Rijeka verwendet wird, obwohl der italienische Begriff Fiume auch heute noch von den Bewohnern der Stadt verwendet wird.

/7/ Vgl. dazu weiter Dubrović, Ervin (2012). From Central Europe to America 1880-1914. Muzej grada Rijeke. Rijeka-New York. S. 116.

/8/ Vgl. dazu Dubrović, Ervin (2012). Op.cit.

/9/ Im Bürgeregister der Stadt Rijeka aus dem Jahre 1857, das heute im Staatsarchiv der Stadt Rijeka bewahrt ist, wird als Geburtsort Triest angeführt. Vgl. dazu auch Lukežić, Irvin (2011). Natalia de Ciotta - ugledna riječka dobrotvorka i njemačka spisateljica. In: Novi Kamov Nr.4. Bd. 41. Jahr XI. Rijeka: Izdavački centar Rijeka. S. 49-74.

/10/ Vgl. dazu weiter Žagar-Šoštarić, Petra/ Lukežić, Irvin (2019). Spuren deutscher Sprache, Literatur und Kultur in Rijeka (Fiume). Vorüberlegungen zu einer Edition des Werkes von Natalia Ritter. In: Möbius, Thomas/ Engler, Tihomir (Hg.): Zwischen Assimilation und Autonomie: neuere Forschungsaspekte zur Kulturgeschichte der deutschsprachigen Minderheit in Kroatien. S. 99121.

/11/ Johann Ritter ist ein erfolgreicher Salpeterhändler und Besitzer zahlreicher Zuckerraffinerien in Görz und Umgebung. Auch war er der Vorsitzende der damals äußerst bekannten Versicherungsanstalt Assicurazioni Generali in Triest, die vor allem im Bereich des Seewesens tätig war. Johann Christian Ritter engagiert sich nicht nur wirtschaftlich, sondern ist auch im öffentlichen Leben äußerst bedeutend: als Kurator der Protestantengemeinde der Schweiz (Comunitá della Confessione Evetica) in Triest. Er gründete auch die evangelische Gemeinde in Görz. Die Nachkommen Ritters erbten außer dem Adelsnamen, ein erfolgreiches Industrieimperium auf den Gebieten von Friaul, Slowenien, Kärnten, Istrien bis hin nach Ungarn und die Slowakei. Nach seinem Tod übernahmen seine Söhne Christian, Hector Julius und Wilhelm die gemeinsame Aufsicht und Leitung der Zuckerraffinerien. Da die Söhne ein reiches finanzielles Erbe erhielten, investierten sie dieses in die Gründung und weitere Entwicklung einer Baumwollindustrie in Podgora neben Görz.
Vgl. dazu weiter Žagar-Šoštarić, Petra/Lukežić, Irvin (2019). Op.cit., S. 99-121.

/12/ Bakar ist ein nur zwanzig Kilometer von Rijeka entfernter Fischerort. Einst war dieser Ort für Thunfisch bekannt, doch nach dem Ausbau einer Koksanlage in Bakarac zu Zeiten Jugoslawiens ist diese Bucht nur noch ein Badeort für Einheimische.

/13/ Edward Bulwer Lytton war ein bekannter Lyriker, Dramatiker und Romancier, welcher der heutigen Öffentlichkeit vor allem anhand des Werks Die letzten Tage des Pompei bekannt wurde.

/14/ Die Familie Scarpa machte sich auch um die Entwicklung des Tourismus in Abbazia/Opatija verdient, weil ihre Villa Angiolina, die sich heute laut zahlreicher Reiseführer in einem der schönsten europäischen Parks befindet, die erste Villa war, die anfing Gäste einzuquartieren. Es waren zunächst enge Freunde und Bekannte der Familie. Später bekam die Villa den Charakter eines Hotels und so zählte Scarpas Villa zu den Anfängen des Hoteltourismus in Opatija.

/15/ Das älteste Hotel am Kvarner befindet sich in Opatija und ist, wie bereits zu Anfang dieses Beitrags erwähnt, von der Familie Scarpa unter dem Namen Villa Angiolina gegründet.

/16/ Pietro Scarpa besaß Anteile dreier Familien-Segelschiffe: Iginia, Paolo und Paolo Maria. Das Segelschiff Iginia, um ein Beispiel hervorzuheben, wird in Fiume 1852 erbaut und hat die Tragmöglichkeit von 325 Tonnen mit einer Schiffsbesatzung von 11 Leuten. Die Anteile an den Segelschiffen teilt er mit seinem Vater und dem Bruder Natalias - Enrico Ritter de Záhony aus Triest. Das Segelschiff Paolo wird auch in Rijeka 1854 gebaut und hat die Tragkraft von 389 Tonnen.

/17/ Das heutige Theater in Rijeka ist 1883 nach Skizzen der deutschsprachigen Architekten Hermann Gottlieb Helmer und Ferdinand Feller gebaut. Dank Adamich (Adamić) wurde am 3. Oktober 1805 das Gemeindetheater (Teatro Comunale) in Rijeka eröffnet; u.a. war er einer der einflussreichsten Männer dieser Zeit. Er war vor allem im wirtschaftlichen, industriellen und politischen Leben der Stadt sehr aktiv, weshalb die heutigen Stadtbewohner diese Zeit in Rijekas Entwicklung geläufig als Die Adamichs Zeit (Adamićevo doba) bezeichnen. Vgl. dazu weiter Moravček, Goran (2012). Adamićevo kazalište u Rijeci. In: Fluminensia. http://www.fluminensia.org/tag/giovanni-de-ciotta.

[Zugriff: 23.01.2018]. S. 1-7).

Ciotta unterstützte auch die Torpedo-Fabrik, die er z.T. gemeinsam mit Robert Whitehead u.a. in Rijeka gründete. Zu erwähnen sei an dieser Stelle 
44

auch, dass Ciotta mütterlicherseits der Enkel des bekannten Adrian Ljudevit Adamich ist. Er ist, wie Natalia, ein großer Wohltäter, der viel in die Ausbildung von Kindern und das Schulwesen investierte. Er war der Meinung, dass jeder Bürger durch Ausbildung zum Guten erzogen werden kann. Schulen und Gesellschaftsräume für Erziehung und Kunst wurden unter seiner Aufsicht gebaut. Vgl. dazu weiter Matejčić, Radmila (1990). Kako čitati grad. Rijeka: ICR. Rijeka.

/18/ Maria Scarpa war die Mezänin der Gesellschaft und Littrow leistete einen großen Beitrag zur lebhaften Entwicklung der österreich-deutschen Kultur in Rijeka.

/19/ Es wird auch Pic-nic geschrieben.

/20/ Der Autor dieser Komödien ist im Tagesblatt $L a$ Bilancia nicht erwähnt. Im DAR (Staatsarchiv Rijeka und der Universitätsbibliothek) ist kein Hinweis zum Autor dieser Werke gefunden. Es besteht jedoch die Annahme (so aus dem Gespräch mit Lukežić am 30.1.2018.), dass diese Werke aus Heinrich von Littrows Feder stammen. Vgl. dazu weiter La Bilancia (1870). III, Nr. 1, 1. I.1870. Societá Pic-nic und La Bilancia (1871). II, Nr. 46, 25. Pic-nic. Prelezioni o trattanimenti durante la quaresima.

/21/ Ein Exemplar dieses Werkes ist in der Universitätsbibliothek in Rijeka gefunden, wo es aufbewahrt ist. Die Geschichte eines Regenmantels und andere hier angeführte Werke wurden bisher nicht gefunden. Man recherchiert zur Zeit danach. Derzeit wird zu diesem Roman eine $\mathrm{Ab}$ schlussarbeit (im Rahmen des Faches Literarisches Übersetzen an der Abteilung für Germanistik an der Universität Rijeka) geschrieben. Die Arbeit widmet sich der Transkription und Übersetzungsproblematik aus der Sicht der heutigen Germanistikstudierenden in Rijeka. Vgl. Ciotta, (von) Natalia (1878). Metamorphosen. Roman in 2 Bänden. Khár \& Wein. Budapest. (Umfang, Band I: S. 207; Band II: S. 192). (Fond Biblioteca civica, Nr. V.D. 21; Universtitätsbibliothek in Rijeka).

/22/ Bekannt ist Brelich auch anhand der Übersetzungen der Werke des ungarischen Literaten Móra Jókaia. Vgl. dazu La Bilancia (1873). Nr. 117, 23. III. 1873. Letteratura und La Bilancia (1873). VI, Nr. 259, 11. XI. 1873. Visita.

/23 Vgl. dazu La Bilancia (1873). Nr. 117, 23. III. 1873. Letteratura.

/24/ Vgl. dazu La Bilancia (1878), Nr 2., 2, giovedí 3. gennaio 1878. Un nuovo romanzo.

/25/ Es geht dabei um einen Reisebericht. Sie notierte und sammelte Eindrücke von der mit ihrem Ehemann unternommenen Ägyptenreise. Einen weiteren Reisebericht verfasste sie noch im gleichen Jahr auf Ungarisch unter dem Titel Fiume-Modrus megye. Vgl. dazu weiter Lukežić, Irvin (2011). Natalia de Ciotta - ugledna riječka dobrotvorka i njemačka spisateljica. In: Novi Kamov Nr.4. Bd. 41. Jahr XI. Rijeka: Izdavački centar Rijeka. S. 4974.

/26/ Vgl. dazu weiter Lukežić, Irvin (2011). Op.cit. S. $49 \mathrm{ff}$.

/27/ Vgl. dazu weiter Lukežić, Irvin (2011). Op.cit.

/28/ Pataky, Sophie (1898) (Bd.1 u. Bd.2). Lexikon deutscher Frauen der Feder. Eine Zusammenstellung der seit dem Jahre 1840 erschienenen Werke weiblicher Autoren. Verlagsbuchhandlung von Carl Pataky. Berlin.

Die Forschungsarbeit zum Werk von Natalia Ritter (Scarpa, de Ciotta) erschwert auch die Tatsache, dass Werke unter einem höchstwahrscheinlich noch weiterem und bisher nicht bekannten Pseudonym (außer R. Enze) geschrieben worden sind.

/29/ An dieser Stelle ist hervorzuheben, dass unlängst (2020) auf Initiative des Museums der Stadt Rijeka eine Publikation zur Geschichte der Marineausbildung in Rijeka und Pula publiziert wurde. Diese wurde allen Offizieren und Professoren gewidmet, die für den Ausbau, den Betrieb und die Entwicklung der Marineakademie in Rijeka verantwortlich waren. Die Sammlung bietet unter anderem, einen Einblick in die Spuren der deutschen Kultur, wobei zum ersten Mal Namen deutschsprachiger Wissenschaftler, Kapitäne, Schriftsteller und Professoren hervorgehoben wurden, die zum Teil diesen hier vorliegenden Beitrag ergänzen. Erwähnenswert ist auch, dass die Naturwissenschaftliche Gesellschaft bzw. der Naturwissenschaftliche Club Fiume eine große Rolle in der gesamten Forschungsarbeit und den Aktivitäten der Marineakademie in Rijeka hatte. Nicht nur Heinrich von Littrow, Natalia Ritter (Scarpa/Ciotta) und Arthur Steinacker, die in dieser Arbeit herangezogen sind, waren wichtig. Weitere deutschsprachige Namen waren bedeutende Mitglieder des Naturwissenschaftlichen Clubs: Peter Salcher, Michael Mayr, Franz Blechschmidt, Karl Kleckler, Josef Koettstorfer, Franz Karl Laufer, Josef Luksch und viele andere, die in diesem Beitrag nicht weiter erwähnt aber derzeit zum Thema Spuren deutscher Kultur, Literatur und Sprache in Rijeka und Umgebung zu untersuchen wären. Vgl. dazu Alebić - Juretić, Ana (2019). Klub prirodnih znanosti. In: Dubrović, Ervin (Hg.): Mornarička akademija. Mornaričko obrazovanje u Rijeci. Muzej grada Rijeke. $42-48$.

/30/ Vgl. dazu Lukežić, Irvin (Handschrift): Od Weimara do Rijeke. Povijest obitelji i životopis riječkog 
bankara i tvorničara Artura Steinackera. S. 61. (derzeit noch unveröffentlicht).

/31/ Fried, Istvan (1990): Das deutschsprachige Bürgertum von Pest-Ofen in den 1840er Jahren. In: UngarnJahrbuch 1990. Verlag Dr. Rudolf Troflenik. München. S. 23-24. Hier zitiert nach Lukežić, Irvin (Handschrift): Od Weimara do Rijeke. Povijest obitelji $i$ životopis riječkog bankara i tvorničara Artura Steinackera. S.61. ff. (derzeit noch unveröffentlicht).

/32/ Es wird in diesem Beitrag nicht tiefgehend über die anderen Familienmitglieder berichtet, (wie z.B. über Arthurs Bruder, Edmund, der ein äuBerst wichtiger deutsch-ungarischer Politiker war und Schwester Irma -Flora Steinacker, die eine in Triest 1847 geborene deutsche- ungarische bekannte Pianistin war). Auch wird der einflussreiche Freundeskreis, in dem die Familie verkehrte, hier nicht weiter thematisiert.

/33/ Vgl. dazu weiter Žmegač, Viktor (2006). Od Bacha do Bauhausa. Povijest njemačke kulture. Zagreb.

/34/ Vgl. dazu Lukežić, Irvin (Handschrift). Od Weimara do Rijeke. Povijest obitelji $i$ životopis riječkog bankara i tvorničara Artura Steinackera. (derzeit noch unveröffentlicht). Vgl. dazu auch weiter Žagar-Šoštarić, Petra/ Kučiš Vlasta (2020). Migration und Multikulturalität in der europäischen Kulturhauptstadt Rijeka mit dem Fokus auf der deutschsprachigen Kultur. (derzeit noch unveröffentlicht).

/35/ Gedichtsband aus dem Jahre 1883 unter dem Titel Von Wien an die Adria nach Triest und Fiume: Reisebilder in gemüthlichen Reimen. Wallishauser. Wien. Obwohl Heinrich von Littrow (1820-1895) in diesem Beitrag nicht tiefgehender veranschaulicht werden kann, weil es inhaltlich den Rahmen sprengen würde, sei hier hervorgehoben, dass sich der deutschsprachige Kapitän nicht nur in seinem Fach als Marineexperte und Kapitän bewiesen hat, sondern auch im Bereich Literatur und Kunst. Er hielt Vorlesungen für die Öffentlichkeit und in seiner Freizeit schrieb er Gedichte, Komödien und Prosa. Sein Schaffen umfasst bedeutende, in deutscher Sprache verfasste und bisher nicht übersetzte wissenschaftliche Beiträge, wie z.B.: Aus dem Seeleben, 1892; Die Marine, 1848; Die Semmeringfahrt, 1883; Deutsches Marine-Wörterbuch; Handbuch der Seemannschaft (1859). Verlag Gerold, Wien.
$\mathrm{Zu}$ seinem literarischen Werk gehören folgende Werke: Seemöven. Gedichte 1857; Von Wien nach Triest, 1863 (Reisebericht); Der Kuss. (Lustspiel); Eine gute Lehre. (Lustspiel); Xanthippe. (Lustspiel). Er schrieb als Korrespondent für Die neue freie Presse, Berliner Tagesblatt, Laibacher Zeitung und La Bilancia. Das Lustspiel Xanthippe ist das einzige ins Kroatische übersetzte Werk des Autors. 1870 publizierte er eine maritime Abhandlung unter dem Titel Fiume in maritimer Beziehung. Diese Abhandlung erschien zweisprachig auf Deutsch und Italienisch und war allen Mitgliedern der Stadtverwaltung gewidmet. Sehr gerne wurde diese Abhandlung von der Öffentlichkeit gelesen, da sie wichtige geschichtliche Tatsachen, in einer dem breiteren Publikum zugeschnittenen Schreibform, an die Öffentlichkeit trug und die Maritimgeschichte Rijekas veranschaulichte. Vgl. weiter Lukežić, Irvin (1994). Heinrich von Littrow. In: Fluminensia. Časopis za filološka istraživanja. Rijeka. S. 1-15. Hier S. 6. und Lukežić, Irvin (1994). Fijumanske priče (Ines Srdoč Konestra). In: Fluminensia. Časopis za filološka istraživanja. Rijeka. S. 178 -179.

/36/ Steinacker, Edmund (1937). Lebenserinnerungen. München. Verlag Max Schick. S. 127.

/37/ Heinrich von Littrow (1883). Von Wien an die Adria nach Triest und Fiume: Reisebilder in gemüthlichen Reimen. Wallishauser. Wien. Zitiert nach Lukežić, Irvin (1994). Heinrich von Littrow. In: Fluminensia. Časopis za filološka istraživanja. Rijeka. 1 - 15. und vgl. weiter dazu Stančić, Mirjana (2013). Verschüttete Literatur. Die deutschsprachige Literatur auf dem Gebiet des ehemaligen Jugoslawien von 1800 bis 1945. Berlin. Wien. Weimar. Vgl. noch weiter zu Heinrich von Littrow, Pederin, Ivan (1987). Heinrich von Littrow kao pjesnik pomoraca $i$ njemačke manjine u Hrvatskoj. ADRAS 1. Split: Zavod za znanstveni i umjetnički rad JAZU.

/38/ Welsch, Wolfgang (1997). Transkulturalität. Zur veränderten Verfassung heutiger Kulturen. In: Irmela Schneider (Hg. et.al). Hybridkultur. Medien. Netze, Künste. Köln. S. 67-90.

139/ Scotti, Giacomo (2008). Ich möchte eine Stadt sein. In: Bačić-Karković, Danijela: Rijeka u priči. Herstomatija književnih tekstova o gradu na Rijeci. Filozofski fakultet. Rijeka. Hier zitiert nach Dienes M. Gerhard (2020) (Hg. et.al.): Europa erlesen Rijeka. Wieser Verlag. Klagenfurt. S. 227-228. 